\title{
Analysis of Lipid Components in Zooplankter Individuals by Reactive Pyrolysis-Gas Chromatography in the Presence of Organic Alkali
}

\author{
Yasuyuki Ishida ${ }^{1}$, Hajime Ohtani ${ }^{1}$, Shin Tsuge ${ }^{1 *}$, Tatsuki Sekino ${ }^{2}$, Masami Nakanishi ${ }^{2}$ and Takashi Kimoto $^{3}$ \\ 1 Department of Applied Chemistry, Graduate School of Engineering, Nagoya University, Furo-cho, Chikusa-ku, \\ Nagoya 464-8603, Japan \\ 2 Center for Ecological Research, Kyoto University, Shimosakamoto, Otsu 520-0105, Japan \\ 3 Research Institute of Oceano-Chemistry, Funabashi-cho, Tenouji-ku, Osaka 543-0024, Japan
}

\begin{abstract}
It has been eagerly requested to develop a highly-sensitive method to characterize extremely minute amounts of natural organic materials occluded in meteorites and/or space dusts in order to confirm the existence of life in the extraterrestrial space. In this article, the reactive pyrolysis-gas chromatography (Py-GC) applied to the analysis of the lipid components contained in every zooplankter individual is introduced for the sake of its potential extension to the characterization of trace amounts of the extraterrestrial organic materials. Here, Py-GC was applied to 1) the discriminative analysis among zooplankter individuals cultured in different food concentrations, and 2) the correlation analysis between the distributions of fatty acid components in the lipids of zooplankter individuals and ingested algae phytoplankton.
\end{abstract}

\section{Introduction}

It has been eagerly requested to develop a highlysensitive method to characterize extremely minute amounts of natural organic materials occluded in meteorites and/or space dusts to confirm the existence of life in the extraterrestrial space. Among various analytical techniques, pyrolysis-gas chromatography/mass spectroscopy (Py-GC/MS) has been utilized as a highly sensitive, rapid and convinient method to characterize not only synthetic polymers but also various biomaterials such as humic substances and microorganisms. Actually, a portable Py-GC/MS system was used for the analysis of organic materials on the surface of Mars in the NASA's Viking Mission in 1976. On the other hand, the reactive pyrolysis technique in the presence of organic alkali, such as tetramethylammonium hydroxide (TMAH), originally developed for the characterization of polar organic materials such as condensation polymers has attracted a great deal of scientific attention in the field of biomaterials since this technique gives simplified pyrograms consisting of the characteristic derivatized peaks of the constituents with much higher sensitivity [1-6].

Received 15 September, 1998

*Address correspondence to: Shin Tsuge
In this article, the reactive $\mathrm{Py}-\mathrm{GC}$ applied to the determination of the lipid contents and their fatty acid compositions of every zooplankter individual[5,6], which has been of interest in the fields of biological science in order to get detailed information about its life history, the structure of the population and population dynamics, will be introduced for the sake of its potential extension to the characterization of the trace amounts of extraterrestrial organic materials. Here, Py-GC was applied to 1) the discriminative analysis among zooplankter individuals cultured in different food concentrations [5], and 2) the correlation analysis between the distributions of fatty acid components in the lipids of zooplankter individuals and ingested algae phytoplankton [6].

\section{Experimental}

\section{Materials:}

Daphnia galeata samples were used as zooplanker samples in this work. Nine cultured samples were used in the study of the discriminative analysis among zooplankter individuals, which were raised under different food concentration. Among these, four Daphnia individuals (Hgroup) were cultured in $10^{6}$ Chlorella cells ml-1 (the high food cultures), yielding individual dry weights from 6 to $25 \mu \mathrm{g}$ each. The other five Daphnia individuals (L-group) ranging between 10 and $15 \mu \mathrm{g}$ were kept without giving any food for five days after being cultured in $10^{6}$ Chlorella cells $\mathrm{ml}^{-1}$.

On the other hand, fifteen plankter samples cultured by using one of three algae species, Chlamydomonas $s p$., Chlorella sp. and Scenedesmus sp., as their food in the laboratory were used for the study of the correlation analysis 


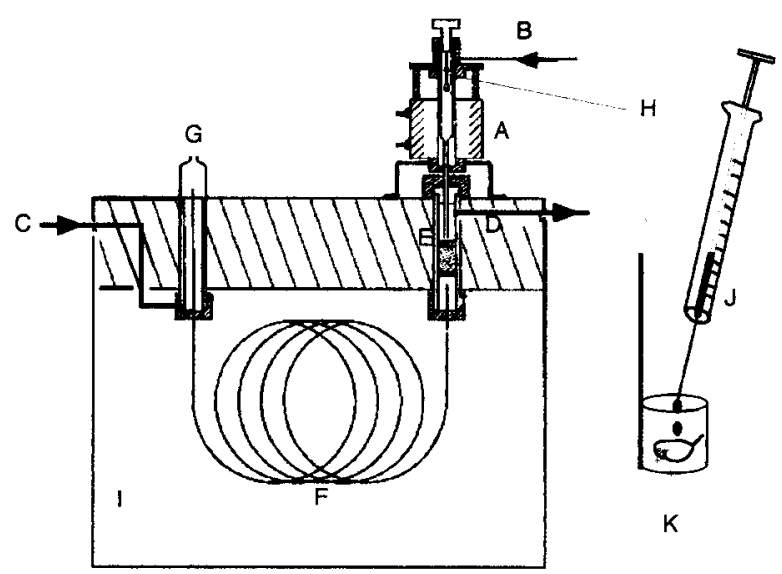

Fig. 1 Schematic flow diagram for high resolution PyGC together with the sampling procedure.

A, microfurnace pyrolyzer; B, carrier gas inlet $(\mathrm{He})$; C, make-up gas $\left(\mathrm{N}_{2}\right)$; $\mathrm{D}$, splitter; E, glass insert tube; F, fused silica capillary column (FSCC); G, flame ionization detector (FID) ; H, platinum sample cup; I, column oven; J, tetramethylammonium hydroxide (25wt \% water solution); $\mathrm{K}$, one zooplankton individual

between the distributions of fatty acid components in the lipids of zooplankter individuals and ingested algae phytoplankton. The food concentration was set at $1 \times$ $10^{6}$ cells ml ${ }^{-1}$ for Chlamydomonas, and $5 \times 10^{6}$ cells ml $^{-1}$ for Chlorella and Scenedesmus. The dry weight of each zooplankter sample ranged from 37 to $62 \mu \mathrm{g}$.

A water solution (25wt.\%) of tetramethylammonium hydroxide (TMAH) supplied by Tama Chemicals Co., Ltd. was used as the reagent for the reactive pyrolysis.

\section{Conditions for Py-GC:}

Figure 1 shows the schematic flow diagram of the measuring system for Py-GC together with the sampling procedure. A vertical microfurnace pyrolyzer (Yanaco GP1018) was directly attached to a gas chromatograph (HP 5890) equipped with a flame ionization detector (FID). One dried plankter individual was weighed by a micro-balance into a small platinum cup. After $2 \mu 1$ of the TMAH solution was added to the same sample cup, it was dropped into the heated center of the pyrolyzer under the flow of helium carrier gas. A relatively low furnace temperature of $400^{\circ} \mathrm{C}$ was used to suppress competitive thermal cleavages. A fused silica capillary column (Hewlett Packard Ultra 1, 25m $\times 0.2 \mathrm{~mm}$ ID) coated with polydimethylsiloxane $(0.33 \mu \mathrm{m}$ film thickness), immobilized by chemical crosslinking, was used for GC. The $50 \mathrm{~mL} / \mathrm{min}$ carrier gas flow rate at the pyrolyzer was reduced to $1.0 \mathrm{~mL} / \mathrm{min}$ at a capillary column by means of a splitter. The column temperature was initially set at $50^{\circ} \mathrm{C}$ and then programmed up to $280^{\circ} \mathrm{C}$ at a rate of 5 ${ }^{\circ} \mathrm{C} / \mathrm{min}$. Identification of the peaks on the pyrograms was carried out mainly using a GC-MS system (JEOL Automass $150)$ with an electron impact ionization $(70 \mathrm{eV})$ source to which the pyrolyzer was directly attached. In order to

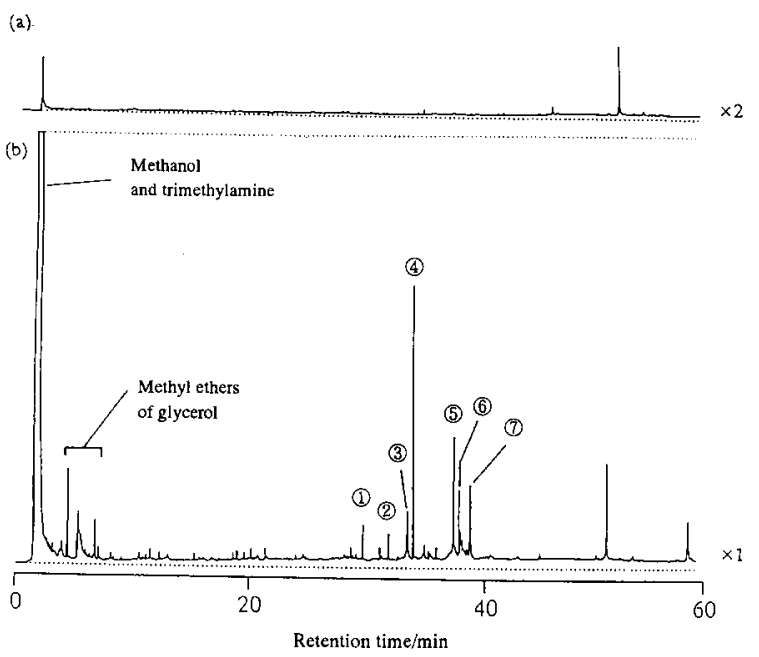

Fig. 2 Typical pyrograms for individual Daphnia sample obtained at $400^{\circ} \mathrm{C}$ without adding TMAH (a) and in the presence of TMAH (b).

Peak assignment: 1, methyl tetradecanoate; 2 , methyl pentadecanoate; 3 , methyl hexadecenoate; 4 , methyl palmitate; 5 , methyl octadecenoate; 6 , methyl stearate;

7 , methyl octadecadienoate

differentiate plankter individuals by considering all the fatty acid components observed on the pyrograms, the resulting data were processed by use of the principal component analysis (PCA) described by Mitsui et al. [7] .

\section{Results and discussion}

Figure 2 shows the typical pyrograms obtained from cultured Daphnia individuals weighing about $11 \mu \mathrm{g}$ for (a) and $21 \mu \mathrm{g}$ for (b) at $400^{\circ} \mathrm{C}$ without addition of TMAH (a) and in the presence of TMAH (b), respectively. Although any characteristic peaks were not observed on the pyrograms obtained without adding the reagent, a series of sharp peaks from 1 to 7 were observed on the pyrogram (b), after the elution of trimethylamine and methanol formed from TMAH solution. These characteristic peaks 1 to 7 were assigned to the methyl esters of saturated and unsaturated $\mathrm{C}_{14}$ to $\mathrm{C}_{18}$ fatty acids, which was formed from the lipid components in Daphnia individual through the reactive pyrolysis in the presence of TMAH. On the basis of the intensities of these characteristic peaks, the lipid contents and the distributions of fatty acid components of every Daphnia individual can be calculated.

Then, reactive Py-GC in the presence of TMAH at 400 ${ }^{\circ} \mathrm{C}$ was applied to differentiate Daphnia individuals cultured in different food concentrations. Table 1 shows the distributions of each fatty acid component and the observed total lipid contents for nine Daphnia individuals. In this table, the distributions of each fatty acid component are similar among these nine individuals regardless of the difference in the food concentration. Here, it is worth noting that the observed total lipid contents (wt\%) for the $\mathrm{H}$ series are about twice as much as those for the L series, although the compositional distribution of the fatty acids 
Table 1 Observed distributions of fatty acid components and total lipid contents for nine Duphnia individuals cultured in different food concentrations

\begin{tabular}{|c|c|c|c|c|c|c|c|c|}
\hline & \multicolumn{7}{|c|}{ fatly acids } & \multirow{2}{*}{ 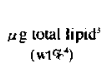 } \\
\hline & 14:0 & $15: 0$ & $16: 0$ & $16: 1$ & $18: 0$ & 18:1 & 18:2 & \\
\hline \multicolumn{9}{|c|}{ relative weight $\%$ : } \\
\hline $\mathrm{H}-1\left(21 \mu \mathrm{g}^{\prime}\right)$ & 4.0 & 3.7 & 25.2 & 12.4 & 7.3 & 30.6 & 16.8 & $1.55(7.4)$ \\
\hline $\mathrm{H}-2\left(\sigma_{\alpha}(\mathrm{g})\right.$ & 7.7 & 3.8 & 23.2 & 10.8 & 7.6 & 34.0 & 12.9 & $0.44(7.3)$ \\
\hline $\mathrm{H}-3(25 / \mathrm{g})$ & 5.0 & 3.5 & 33.0 & 12.6 & 6.7 & 24.2 & 15.) & $2.12(8.5)$ \\
\hline $\mathrm{H}-4(11 \mu \mathrm{g})$ & 1.9 & 1.8 & 28.9 & 9.9 & 7.9 & 28.0 & 21.6 & $0.98(8.9)$ \\
\hline $\mathrm{L}+\mathrm{I}(14 \mu \mathrm{g})$ & 4.1 & 3.2 & 29.9 & 9.8 & 10.4 & 30.3 & 12.3 & $0.45(3.2)$ \\
\hline $\mathrm{L}-2\left(15_{\mu \mathrm{g}}\right)$ & 5.6 & 3.0 & 21.2 & 12.5 & 7.2 & 34.5 & 16.0 & $0.61(4.1)$ \\
\hline$L^{-3}(13 \mathrm{ug})$ & 3.9 & 2.2 & 24.4 & 14.8 & 8.4 & 31.1 & 15.2 & $0.50(3.8)$ \\
\hline $\mathrm{L}-4(11) \mu \mathrm{g})$ & 3.4 & 2.5 & 28.4 & 11.7 & 8.7 & 29.8 & 15.5 & $0.40(4,0)$ \\
\hline L-5 $(13 \mu g)$ & 4.0 & 3.8 & 25.6 & 11.2 & 6.2 & 33.5 & 15.7 & $0.77(5.9)$ \\
\hline
\end{tabular}

1 dry weight measured by microbalance

2 relative weight \% among the observed fatty acid components.

3 total lipid ( $\angle \mathrm{g})$ was estimated assuming that all the fatty acids exist in triglyceride form in Daphnia sample

4 total lipid wt\% was calculated against dry weight of each Daphnia sample

are almost the same between $\mathrm{H}$ and $\mathrm{L}$ series.

Then the PCA was carried out in order to differentiate the nine Daphnia individuals considering all the fatty acid components observed on the pyrograms. The observed peak intensities for methyl esters of fatty acids normalized by sample weights were used as the data base after being reformed to deviation values to equalize the weight. The contributory rates (dispersion) of the $1 \mathrm{st}, 2 \mathrm{nd}$ and $3 \mathrm{rd}$ principal components obtained were 78.6, 14.5 and 3.3\%, respectively. Figure 3 shows the 1 st principal component scores for the nine Daphnia individuals. In this figure, only the 1st principal component was used to differentiate Daphnia individuals because the other principal components reflected the difference in the nutritional status of individuals to the less extents. As shown in this figure, these Daphnia individuals are clearly divided into two groups according to the food concentration in which each individual was cultured. This result indicate that the 1st principal component score can be used as a good measure of food concentration for each Daphnia individual.

Then, reactive Py-GC was applied to the correlation analysis between the distributions of fatty acid components in the lipids of zooplankter individuals and ingested algae phytoplankton. Figure 4 shows the typical pyrograms obtained from ca. $100 \mu \mathrm{g}$ of Chlamydomonas (a) and Daphnia individual weighing about $11 \mu \mathrm{g}$ cultured with Chlamydomonas (b) at $400^{\circ} \mathrm{C}$ in the presence of TMAH. On both pyrograms, the methyl esters of saturated and unsaturated $\mathrm{C}_{14}$ to $\mathrm{C}_{18}$ fatty acids resulted from the reactive pyrolysis are observed after the elution of methanol and trimethylamine formed from excess amount of the reagent. Furthermore, Py-GC measurements of other Daphnia and algae samples also yielded the similar pyrograms, on which

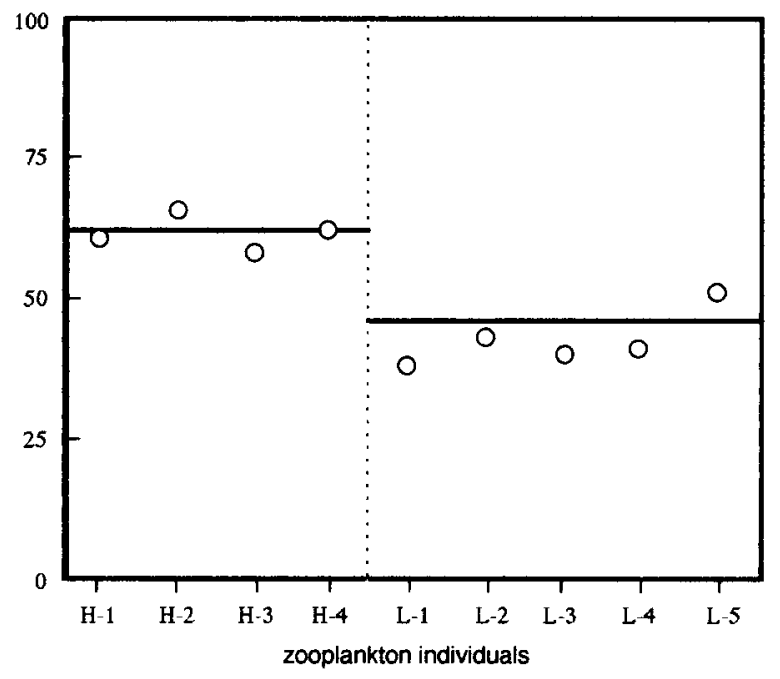

Fig. 3 The 1st principal component scores for nine Daphnia individuals cultured in the different food concentrations.

H-group: cultured in $10^{6}$ Chlorella cells $\mathrm{ml}^{-1}$;

L-group: cultured as H-group, then kept without food for five days.
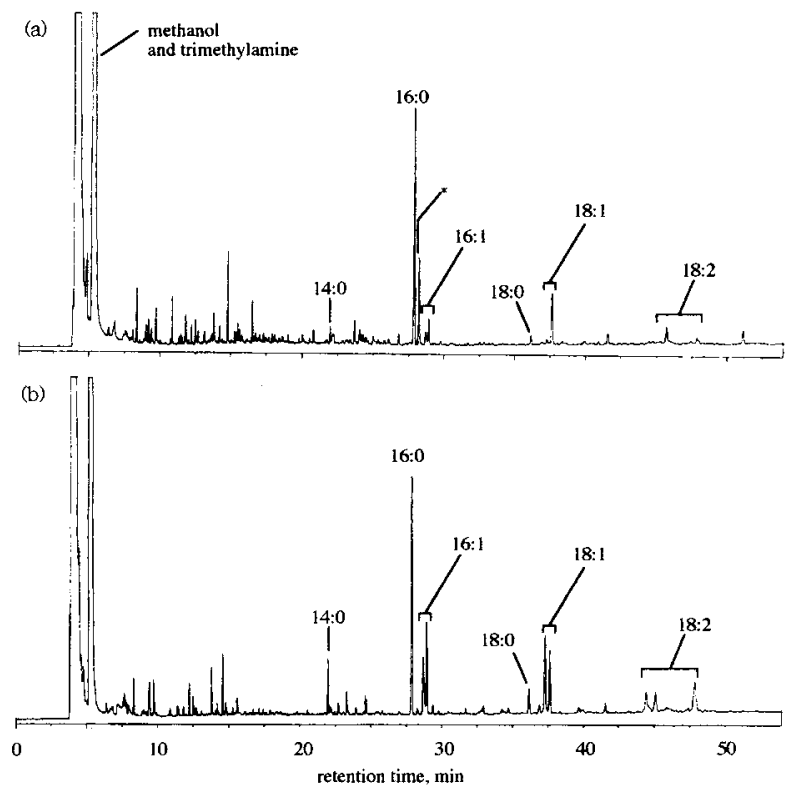

Fig. 4 Typical pyrograms of plankton samples at $400^{\circ} \mathrm{C}$ in the presence of TMAH

(a) ca. $100 \mu \mathrm{g}$ of Chlamydomonas sample

(b) a single Daphnia sample cultured with Chlamydomonas

* This peak refers to one of the pyrolytic components of cellulose. 
Table 2 Observed chemical composition of Daphnia individuals and ingested algae samples

\begin{tabular}{|c|c|c|c|c|c|c|c|c|}
\hline \multirow{8}{*}{$\begin{array}{l}\text { Daphria cultured by } \\
\text { Chiamydomonas }\end{array}$} & \multicolumn{7}{|c|}{ Fatty acids } & \\
\hline & 14:0 & $16: 0$ & 16:1(cis-7) & 16:1(cis-9) & $18: 0$ & 18:1(cis-9) & 18: 1(cis-11) & \\
\hline & \multirow[b]{2}{*}{5.53} & \multicolumn{4}{|c|}{ Compositions (mol $\%$ ) } & \multirow[b]{2}{*}{16.1} & \multirow[b]{2}{*}{11.7} & \multirow{2}{*}{$\begin{array}{l}\text { total } \\
100\end{array}$} \\
\hline & & 37.1 & 11.2 & 13.7 & 4.59 & & & \\
\hline & 4.98 & 33.1 & 13.0 & 13.2 & 5.07 & 19.2 & 11.5 & 100 \\
\hline & 4.86 & 32.0 & 12.4 & 13.7 & 6.00 & 19.6 & 11.4 & 100 \\
\hline & 5.15 & 32.9 & 11.9 & 15.8 & 5.76 & 17.0 & 11.4 & 100 \\
\hline & 5.33 & 37.5 & 10.6 & 16.6 & 3.85 & 14.5 & 11.5 & 100 \\
\hline \multirow[t]{2}{*}{ Mean values } & 5.17 & 34.5 & 11.8 & 14.6 & 5.05 & 17.3 & 11.5 & 100 \\
\hline & 3.45 & 63.1 & 5.19 & 6.77 & 2.71 & 1.87 & 16.8 & 100 \\
\hline \multirow[t]{2}{*}{ Chiamydomonas } & 3.43 & 62.7 & 5.48 & 6.77 & 2.77 & 2.09 & 16.8 & 100 \\
\hline & 2.89 & 66.6 & 5.94 & 6.98 & 2.10 & 1.91 & 13.6 & 100 \\
\hline \multirow[t]{2}{*}{ Mean values } & 3.26 & 64.1 & 5.54 & 6.84 & 2.53 & 1.96 & 15.7 & 100 \\
\hline & 2.91 & 26.3 & 17.6 & 8.59 & 8.59 & 27.0 & 8.98 & 100 \\
\hline \multirow{4}{*}{$\begin{array}{l}\text { Daphnia cultured by } \\
\text { Chlorella }\end{array}$} & 3.66 & 28.3 & 19.7 & 8.31 & 8.41 & 23.5 & 8.03 & 100 \\
\hline & 3.24 & 26.8 & 18.0 & 8.88 & 6.60 & 27.3 & 9.27 & 100 \\
\hline & 3.44 & 27.8 & 17.3 & 8.55 & 6.14 & 27.1 & 9.58 & 100 \\
\hline & 3.14 & 27.0 & 17.9 & 10.1 & 6.68 & 25.6 & 9.50 & 100 \\
\hline \multirow[t]{2}{*}{ Mean values } & 3.28 & 27.2 & 18.1 & 8.89 & 7.28 & $26-1$ & 9.07 & 100 \\
\hline & 1.98 & 34.1 & 10.8 & 2.27 & 2.25 & 39.6 & 9.03 & 100 \\
\hline \multirow[t]{2}{*}{ Chlorella } & 1.73 & 34.6 & 10.9 & 2.34 & 2.02 & 39.4 & 8.99 & 100 \\
\hline & 1.62 & 33.5 & 10.3 & 2.11 & 1.44 & 40.9 & 10.1 & 100 \\
\hline Mean values & 1.75 & 34.1 & 10.7 & 2.24 & 1.90 & 40.0 & 9.37 & 100 \\
\hline \multirow{5}{*}{$\begin{array}{l}\text { Daphnia cultured by } \\
\text { Senedesmus }\end{array}$} & 3.01 & 27.5 & 17.3 & 4.34 & 4.99 & 38.0 & 4.75 & 100 \\
\hline & 2.75 & 26.8 & 16.8 & 4.03 & 4.88 & 40.5 & 4.27 & 100 \\
\hline & 3.01 & 26.5 & 17.5 & 4.01 & 4.84 & 39.6 & 4.55 & 100 \\
\hline & 2.96 & 27.0 & 17.5 & 4.03 & 4.54 & 39.9 & 4.07 & 100 \\
\hline & 2.97 & 28.0 & 17.6 & 4.27 & 4.51 & 38.4 & 4.15 & 100 \\
\hline \multirow[t]{2}{*}{ Mean values } & 2.94 & 27.2 & 17.3 & 4.14 & 4.75 & 39.3 & 4.36 & 100 \\
\hline & 0.84 & 45.0 & 7.41 & & 1.21 & 44.3 & 1.24 & 100 \\
\hline \multirow[t]{2}{*}{ Senedesmus } & 0.67 & 36.9 & 5.54 & - & 1.34 & 54.3 & 1.31 & 100 \\
\hline & 0.81 & 44.0 & 6.65 & - & 1.22 & 45.9 & 1.36 & 100 \\
\hline Mean values & 0.77 & 42.0 & 6.53 & & 1.26 & 48.2 & 1.30 & 100 \\
\hline
\end{tabular}

the series of fatty acid methyl esters from 14:0 to 18:2 were clearly observed. Here the methyl esters of 18:2 components were not used for the estimation of the fatty acid compositions because of relatively poorer reproducibility of their relative peak intensities.

Table 2 shows the observed chemical compositions from 14:0 to 18:1 obtained for a single individual measurement of fifteen Daphnia samples and three repeated measurements for each algae samples obtained by the reactive Py-GC. These data suggest that the observed fatty acid compositions of every Daphnia sample have close correlations to those of the ingested algae sample. For example, for Daphnia samples cultured with Chlorella and Scenedesmus, the relative abundances of 16:0 and 18:1(cis9) are significantly higher than those of other fatty acid components reflecting those for the ingested algae phytoplankton samples. Similarly, the significantly high abundance of 16:0 in Chlamydomonas is preserved in corresponding Daphnia samples. Nevertheless, the positive and/or negative discrepancies between the observed fatty acid compositions for a given Daphnia and the corresponding algae used as the food imply fairly strong contribution of isomerization, degradation and/or elongation of a part of fatty acid components during digestion in Daphnia individuals after intake by the zooplankter.

Therefore, in order to take all the observed compositional information into consideration, the PCA was applied to the observed compositional values for the evaluation of the relationships between a given Daphnia 
group and the corresponding algae samples used as the food. Figure 5 shows the two dimensional visualization of the first and second principal component scores (PC1 and $\mathrm{PC} 2$, respectively). These statistical data were calculated from the observed fatty acid compositions estimated from a single measurement for Daphnia individuals and a single measurement for algae samples shown in Table 2. In this figure, the plots for algae samples are discretely separated into three groups according to the species, while those for Daphnia samples are also clearly separated into the other three groups reflecting the algae species used as food. Furthermore, it is interesting to note that the score plots for Daphnia individuals are shifted in parallel by almost same increment compared to those for ingested algae samples. This fact suggests that lipid biosynthesis such as isomerization and/or elongation of fatty acid components occurs in a similar ratio for these Daphnia individuals independently of the species of ingested algae phytoplankton. These results indicate that the fatty acid compositions of Daphnia individuals used in this work are significantly reflected in those of their food although isomerization and/or elongation of fatty acid components occur during digestion to some extents.

As described above, reactive Py-GC in the presence of organic alkali can be used as a rapid, convenient and highly sensitive method to evaluate total lipid content and the distribution of fatty acid composition in zooplankter individuals weighing in $\mu \mathrm{g}$ order without using any pretreatment. Furthermore, this technique is also expected to offer a new method to characterize the trace amounts of the extraterrestrial bioorganic materials and microorganisms occluded in meteorites and space dusts.

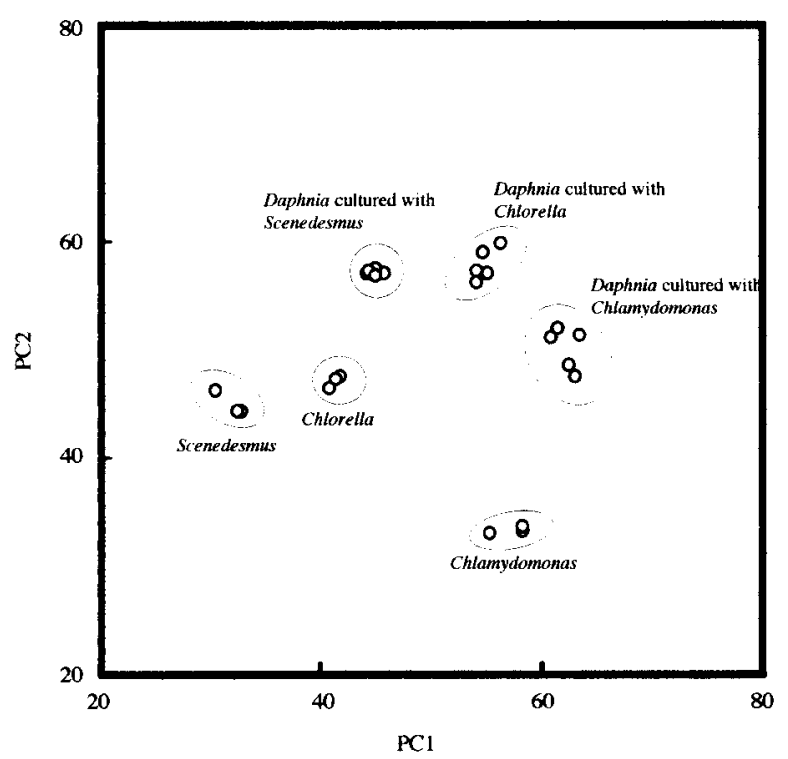

Fig. 5 First and second principal component scores for each Daphnia sample and ingested algae sample

\section{References}

1 Challinor, J. M. (1989), J. Anal. Appl. Pyrolysis, 16, 323.

2 Challinor, J. M. (1991), J. Anal. Appl. Pyrolysis, 20, 15.

3 Ohtani, H., Fujii, R., Tsuge, S. (1991), J. High Res. Chromatogr., 14, 388.

4 Ishida, Y., Ohtani, H., Kato, T., Tsuge, S., Yano, T. (1994), Tappi J., 77, 177.

5 Ishida, Y., Isomura, S., Ohtani, H., Tsuge, S., Sekino, T., Nakanishi, M., Kimoto, T. (1996), Analyst, 121, 853.

6 Ishida, Y., Yokoi, H., Isomura, S., Ohtani, H., Tsuge, S., Sekino, T., Nakanishi, M., Kimoto,T. (1998), J. Chromatogr. B, 716, 39.

7 Mitsui, T., Okuyama, S., Fujimura, Y. ( 1991) Anal. Sci., 7, 941. 